\title{
The characteristics of prototypical occupational identities: a grounded theory of four occupations
}

\author{
Julia Yates*, \\ City, University of London \\ Julia.yates.1@city.ac.uk \\ Sharon Cahill, \\ University of East London \\ s.cahill@uel.ac.uk \\ * corresponding author
}

\begin{abstract}
Evidence suggests that occupational prototypes have an impact on career decisions yet literature provides only a limited understanding of the nature of these prototypes. This paper presents a small-scale grounded theory study to explore the prototypes of four occupations conceptualised by psychology undergraduates. Data were collected from 24 psychology undergraduates through four focus groups. Participants were asked to evoke a typical member of each of four occupational groups and describe their prototype in detail. A classic grounded theory analysis identified the characteristics which were symbolised by the features of the prototypes and resulted in eight dimensions: warm, energetic, fun, intelligent, conventional, highbrow, successful and cool. The discussion explores the dimensions in the light of existing literature and suggestions are offered for practice.
\end{abstract}

Key words: occupational prototypes; occupational identities; career practice; grounded theory; self to prototype matching

\section{Introduction}

This paper describes a study aimed at furthering our understanding of occupational prototypes. The study offers an empirically derived theoretical framework of the characteristics of psychology undergraduates' perceptions of four prototypical occupational identities. Occupational prototypes are representations or images of a stereotypical member of an occupational group, and their influence on career decisions has been well documented. Their significance is highlighted in Gottfredson's influential theory of circumscription and compromise (Gottfredson, 1981, 2002) and in identify-fit theory (Peters, Ryan, Haslam \& Fernandes, 2012). Their role in the decision-making processes of those choosing against careers in science, technology, engineering and maths (STEM) subjects has also been widely examined (for example, Archer et al. 2010; Cheryan, Plaut, Handron \& Hudson, 2013; Hannover \& Kessels, 2004). Yet the literature provides a limited account of the nature of these prototypical occupational identities, a dearth of theory to account for them, and a lack of 
practical tools for career counsellors which can facilitate the exploration of occupational prototypes in career conversations.

In the next section, we will introduce occupational prototypes, highlighting the role of symbols in conceptualisations of prototypes, and their influence on career decision making, and will expose the limitations of the theoretical basis within this field. The methodology and findings are then described and a discussion integrates the theory of prototypes in the light of existing literature and suggestions for the practical implications. In keeping with the classic grounded theory methodology used in this study, some new literature, relevant to the findings of the study will be introduced in the discussion section.

\section{Theoretical Background}

The importance of identity in career development has become increasingly mainstream in the career literature (Savickas et al., 2009) with an acknowledgment that career decisions are about 'Who will I be?' as much as 'What will I do?'. This identity approach to career development has been widely documented (e.g., Ibarra \& Barbelescu, 2010; Meijers \& Lengelle, 2012). In making an occupational choice, an individual also makes the choice to join the particular occupational group and perhaps embrace the social identity of that group (Guichard, 2005). As such the perception of the occupational identity is particularly pertinent to our understanding of occupational choice.

Stereotyping is an efficient cognitive procedure which enables us to interpret identities (Lippman, 1991). Stereotypes are cognitive shortcuts which allow people to make sense of complex and overwhelming information, but they can also serve to perpetuate social inequalities (Schneider, 2004). Evidence suggests that they can be reasonably accurate, but gloss over individual differences (Hilton \& Von Hippel, 1996; Jussim et al., 2009). The information which feeds into the development of stereotypes is thought to come from both shared experiences, such as the influence of peers and parents (Castelli, Carraro, Tomelleri \& Amari, 2007) or television (Mastro, Behm-Morawitz \& Kopacz, 2008), and individual experiences (Haslam \& Wilson, 2000). The influence of both shared and individual experiences means that whilst there may be some commonality between different people's stereotypes, there will be differences too.

Prototypes are a form of stereotype in which the features and attributes of the stereotype of a particular group are combined and represented in a single conceptualisation of an identity (Rosch \& Mervis, 1975). An occupational prototype is thus a mental representation of a typical member of a particular occupation. Occupational prototypes are based on stereotypical thinking but represented as an imagined person (a stereotypical social worker, or a stereotypical builder, for example). Occupational prototypes encompass a wide range of types of information about the occupations and those who inhabit them, including appearance, emotions, cultural beliefs, values and lifestyles (MacKinnon \& Langford, 1994; Yates \& Cahill, 2018). As occupational prototypes derive from stereotypical features, they comprise elements which are common to others' prototypes and aspects which are personal to each individual's conceptualisation of the prototype. Given that the literature highlights that prototypes consist of a wide range of features and these can be conceptualised as symbolic objects, an 
understanding of these symbols may serve to shed light on the meanings of these occupational prototypes.

Making sense of the world of work is no mean feat, and objects can help to enhance our understanding. In every encounter which feeds our world-of-work knowledge, we can become aware of the clothes, homes, hobbies and choices of people within particular occupational groups. To make sense of these, we isolate, identify and catalogue them. Each of these social objects becomes a symbol, laden with meaning. The symbols have no intrinsic link to their meaning, but the meaning emerges from the way that we see people responding to the object. These symbols have a key role in the way that we make meaning from our surroundings (Charon 2010, p.59).

These objects are used by individuals to communicate identities, and it is this, in part, that makes them relevant to our own career decision making. The objects tell us something about the occupational identities of these people and are therefore key to our perceptions of the world of work. Messages about our identity are conveyed to others through many different kinds of objects (Charon 2010). Symbols in a work context include artefacts, language, rituals, stories and myths, and they are described as a language which communicates identity and status (Pratt \& Rafaeli, 2001). Objects are thus used as cognitive heuristics which allow individuals to navigate, make sense of and represent complex phenomena in a manageable and accessible way (Pratt \& Rafaeli, 1997).

As highlighted by a number of scholars (Kocoglu, Akgun \& Keskin, 2016; Pratt \& Rafaeli, 2001; Strati, 1998) the research on work-related symbols has been largely descriptive, and there have been limited attempts to provide a more conceptual analysis or theory of the symbols, or to offer frameworks to elucidate the meanings behind the symbols which could be used as a practical tool.

\section{Occupational prototypes and career development}

It has long been acknowledged that stereotypes and prototypes have an impact on career decisions. Gottfredson (1981) demonstrated the influence of gender and prestige in prototypes, and since then there has been considerable empirical evidence of the impact of the gender of occupational stereotypes on career choice (Archer et al., 2010; Gadassi \& Gati, 2009). Self-to-prototype matching is an approach to career decision making in which a choice is made on the basis of the degree to which an individual's self-concept aligns with their prototype of a particular occupation: an individual is more likely to opt to study computer science if they see themselves as similar to their idea of a typical computer scientist. This approach conceptualises prototypes as highly differentiated, explaining the influence not only of gender and class, but other prototypical features such as personality, appearance and hobbies. The evidence that this approach has an impact on participants' career decision making is growing (for example, Cheryan \& Plaut, 2010; Hannover \& Kessels, 2004; McPherson, Park \& Ito, 2018; Ryan, 2014; Starr, 2018), but there are three notable limitations within this literature.

First, studies have tended to focus on the degree to which participants' self-concepts have differed from a commonly held view of a single occupational prototype (Ehrlinger et al., 2018). 
We know that people's occupational prototypes are based on a combination of societal experiences and personal experiences, so whilst occupational prototypes have shared elements, they also have individual ones (Haslan \& Wilson, 2000); the understanding gained through self-to-prototype matching research which explores the influence of just the shared aspects and not the individual aspects of the prototypes can then only ever be partial. The second limitation of the body of research is its almost exclusive focus on STEM jobs. The impetus to understand the under-representation of women in STEM fields means that this is a well-researched area, but our understanding of the self-to-prototype matching approach would be enhanced if empirical research centred on a broader range of occupational areas. The final shortcoming lies in the limited practical application of self-to-prototype matching. Whilst research, policy and practice all address the issues of gender prototypes, scant advice is offered for career guidance professionals interested in using their understanding of self-toprototype matching and its more detailed conceptualisations of occupational prototypes.

The restricted reach of self-to-prototype matching, both in research and practice, could be a consequence of our incomplete understanding of occupational prototypes. With research examining just the shared aspects of one occupational prototype at a time, and a limited theoretical underpinning to the approach, it is perhaps not surprising that the evidence base has grown slowly and has not been recognised widely.

This study begins to close these gaps, offering a small-scale grounded theory based on the perceptions of four occupational prototypes described by 24 psychology undergraduates. We aimed to identify the characteristics symbolised by the features of the occupational prototypes. The research question for the study was: What are the characteristics symbolised by the features of four occupational prototypes evoked by psychology undergraduates? A grounded theory approach was used. Participants were invited to describe their individual versions of the occupational prototypes, and the characteristics symbolised by the features of these prototypes form the basis of the theory. The theory therefore extends our understanding of the nature of occupational prototypes, exploring a range of different features and the characteristics they represent.

\section{Method}

\section{Research Design}

Developed by Glaser and Strauss (1967), grounded theory (GT) is a method for inductively generating theory in which the analysis of data results in a theory that is grounded in the words, behaviours and perceptions of the participants (Glaser, 1992). Despite the painstaking coding and challenges of developing a theory without reference to existing literature (Hussein et al., 2014), GT is now accepted as a rigorous approach to qualitative research in psychology (Willig, 2013), and is widely used in career development research (e.g., Boychuck, Lysaght \& Stuart, 2018; Jung \& Young, 2019). Its systematic approach to data analysis provides academic rigour, and its position, bridging quantitative and qualitative traditions in psychology research (Charmaz \& Henwood, 2013) allows researchers to 'increase their efficiency and effectiveness in gathering useful data and in constructing focused analyses'(p.241). 
Beyond these advantages, a GT analysis was thought to be particularly suitable for this study. As mentioned earlier, there is acknowledgement within the research community that occupational prototypes have some impact on career choices. There have, however, been no attempts to integrate these results into a coherent framework, or to apply existing theories to understand or explain the results. We propose that this atheoretical approach to the research on occupational prototypes may have contributed to the limited impact that it has had on the career decision-making literature and practice. GT is a methodological approach thought to be particularly useful in 'investigations of relatively unchartered waters' (Stern 1980, p.20), and is considered the 'method of choice when a descriptive theory is not available' (Crooks 2001, p. 12). It was therefore considered to be suitable for this study.

GT has undergone a number of theoretical splits. Fernandez (2012) identified four key models of GT which are most commonly used within qualitative research: Classic Grounded Theory (Glaser, 1978), the Strauss and Corbin version (1990) known as qualitative data analysis, constructivist Grounded Theory (Charmaz, 2013) and feminist Grounded Theory (Wuest, 1985). This study has used the Glaser method of GT (1978), which has come to be known as Classic Grounded Theory (CGT). Glaser emphasised the emergent nature of the theory and stresses that the theory can only explain the phenomenon under study $(1992,2003)$, arguing that too much emphasis on systematic coding will detract from insightful meaning. The basic principles of open coding, axial coding, theoretical sampling and abstraction are key to the process, but the more fixed coding processes as developed by Strauss and Corbin (1990) are not applied. CGT was chosen as the method of analysis for this study as it is believed that the more flexible approach of CGT allows for greater creativity and for a clearer link between the theory and data (Bowen 2005, Clark \& Lang, 2002; Davis, 1996; Holton, 2007; Schreiber, 2001).

Strauss and Corbin (1990) described theories as either substantive (i.e. explaining just the current phenomenon) or formal (generalisable). The GT described in this study is a substantive theory. It is common for GT to lead to substantive theories as the theories are closely linked to the data of one particular study. It is important to emphasise that a grounded theory is not intended to reflect an absolute truth, but is a modifiable and tentative explanation of the phenomenon.

\section{Participants}

Participants for this study were drawn from students on psychology programmes at a university in London. This cohort was chosen as they would not be currently working in the occupations under discussion, but might be assumed to have an interest in them. Once ethical approval was granted, an email was sent, inviting all undergraduates to take part in the study; 48 students offered to participate and 27 who had not worked in or close to the occupations under discussion were selected and invited to the focus groups. Three students were not able to attend the focus groups, resulting in a total of 24 participants. The students were all female and ranged in age from 20 to 42 years. They were all enrolled in the BSc programme in psychology at the university. Most of the students were British (20), but one participant was French, one German, one Portuguese and one from the USA.

\section{Procedure}


Focus groups are a widely used form of data collection for qualitative research within the social sciences (Hyde, Howlett, Brady \& Drennan, 2005). A focus group allows an insight into the group's collective experiences, and the discussion is structured to allow as much freedom and interaction as possible, whilst maintaining the focus on the topic (Bagnoli \& Clark, 2010). In a focus group, the expectation is that the group dynamic and the interaction between group members will allow the individuals to explore and clarify their own views more effectively than they might in a one-to-one interview. The themes are actually constructed within the group setting (Gough, Fry, Grogan \& Conner, 2009) and in this way focus groups are able to capture something of the essence of the 'social construction of experience' (Kitzinger, 1994, p. 172).

The focus group data can reveal information about the group itself, its dynamics and its shared and unique culture (Sherriff, Gugglberger, Hall \& Scholes, 2014) whilst simultaneously generating insights within each group member, as comments and responses from others spark off new thoughts in participants. (Gough et al., 2009; Warr, 2005). Focus groups are thought to be particularly suitable for exploring new topics (Sim 1998) as a significant volume of data can be collected efficiently; as such they were considered suitable for this research. Moreover, the principal methodological challenges presented by focus groups (Hennick, 2007) are that individuals may not feel comfortable voicing their personal views, and that issues of a sensitive nature are not generally thought to be suitable topics. Neither of these issues was considered significant within this study, as the value of the findings would not be predicated on full disclosure of each participant's views, and the topic was not thought to be a particularly delicate one.

Data were gathered through a series of four focus groups, with six participants in each, and the discussions were audio recorded and transcribed verbatim. Focus groups typically have 6 to 12 participants (Krueger, 1994). This is considered sufficient for a lively discussion, but small enough to ensure that everyone gets an opportunity to voice their views. Participants were asked to conjure up an image of a 'typical member' of a particular occupational group and were asked to describe this prototype in detail, focusing on a wide range of features including appearance, personality, attitudes and behaviour.

Four occupations were discussed, two in each of the four focus groups, and the order in which the occupations were presented to the groups was varied to avoid bias from order effect. As there were four different occupations under scrutiny, it was thought that a minimum of four focus groups was needed. This would then ensure that an impact on the validity of the data through order effect was avoided. Each occupation was discussed first on one occasion, and each discussed by two or three of the groups overall. Whilst it would have been interesting to have each occupation discussed in each focus group, we wanted in-depth discussions and it was thought that this would be likely to result in focus groups which were long and risked fatigue in participants, or which produced less rich descriptions of each prototype. The focus groups each lasted approximately 2 hours. To identify occupations which participants were likely to be familiar with, the most popular graduate-level occupational choices of recent psychology undergraduates at the university were identified using the destinations data from the previous cohort of graduates (HESA, 2014). The occupations identified were: primary teacher, social worker, occupational psychologist and clinical psychologist. 
A warm and informal tone was set for the discussions, instructions were clear and plenty of time was allowed for questions (Hyde at al. 2005). Issues of confidentiality and mutual respect were stressed (Tolich, 2009). An interview guide was developed to ensure that the discussion was sufficiently wide-ranging to generate data that would answer the research questions (Hyde, et al., 2005). The questions were based on previous literature which covered occupational prototypes, and in the absence of much published work, our own intuition of the kinds of topics which might lead to insightful conversations, and were developed expressly to answer the research questions. Questions included: 'Tell me about your prototype.', 'What matters to them?', 'What would they wear to work?', 'Where would they go on holiday?' and 'How would you describe their personality?' The analysis of data from each focus group took place prior to the next group scheduled in accordance with CGT practice (Glaser, 1992) and theoretical saturation was reached after three focus groups (Strauss \& Corbin, 1990). A fourth focus group was run to avoid bias due to order effect by ensuring that each occupation was discussed first once.

\section{Data Analysis}

In CGT the researcher sticks faithfully to the data and limits the impact that other influences such as existing literature may have (Hernandez, 2008). The CGT researcher aims to base assumptions only on the data, and tries to minimise the influence that their own preconceived knowledge has on the coding or development of theory. Proponents of this approach acknowledge that it is not possible to eliminate these influences altogether, and that objectivity (whilst arguably desirable) is not a realistic goal for the researcher. The success of reflexivity is contingent on the self-awareness of the researcher and as such will inevitably be partial (Cutcliffe, 2003). Nevertheless, reflexivity is an important part of the process and every effort was made during the analysis of this study to follow where the data led (Lowe, 2005). Memos were used throughout the research process as a mechanism for noting the our own responses to the data and the emerging theory, and the content of the memos informed the development of the theory (Charmaz, 2013).

The data analysis process followed in this study followed Glaser's (1965) four stages: 1) comparing incident applicable to each category, 2) integrating categories and their properties, 3) delimiting the theory and 4) writing the theory. The stages will now be described and illustrated with examples. Whilst the stages are covered here in turn, it is important to acknowledge that in practice it was not (and nor should it have been) quite as linear; as Christiansen suggests (2007), the stages should occur 'simultaneously or serendipitously'(p. 49). The onset of each stage of the analysis took place in this chronological order, but the earlier stages remained in operation throughout the process of analysis.

\section{Comparing incidents applicable to each category}

The starting point for the analysis is open coding, in which every 'incident', or comment is coded in as many ways as can be conceived. The codes generated were interpretations of the underlying meaning of the features the participants described: the features of the prototypes 
described in the focus groups were symbols, and the coding articulated the constructs symbolised.

The information which allowed the identification of the codes came from two sources: the our own individual responses as researchers and explicit comments made by the participants: 'those which he [the researcher] had constructed himself; and those that have been abstracted from the research situation' (Glaser, 1965, p 438). For example, one participant described her prototype of a clinical psychologist as wearing Birkenstocks, and said explicitly that these were 'unconventional': Birkenstocks were thus coded as 'unconventional'. The same participant suggested that this clinical psychologist would drink red wine with meat and white wine with fish. She offered no explicit explanation of what this behaviour might symbolise, but, drawing from our own personal responses, it was proposed in the coding that this might symbolise someone with a high status, possibly educational status or cultural status: someone who knows what colour wine 'ought' to go with certain foods.

The grounded theory researcher should engage in constant comparisons during the coding process, comparing codes with codes: as the feature of 'dungarees', for example, which was described as part of one of the primary teacher prototypes, was coded in the line-by-line analysis, it was considered in the light of other fashion items which had been described in the focus groups (such as a sharp suit, or Birkenstocks), other items which might be symbols of fun (such as enjoying karaoke) or other items which might be symbols of a serious character (such as watching documentaries). This process of constant comparison allowed for the features and symbols to be categorised, to identify features which they have in common and those which distinguish them from each other.

It was during this process of constant comparison that the properties of the theory began to take shape. As the different features and the characteristics of the features were considered, it became clear that there were certain underlying elements which they shared. For example, watching comedy programmes, having a laugh with mates, wearing bright colours, all emerged as symbols of fun.

Throughout the process of analysis memos were written, allowing us as researchers to capitalise on our first thoughts and reactions. Researchers are advised to write memos down as soon as they can after the idea has emerged and this makes sure that even the vaguest of notions, fluttering through the consciousness is recorded (Christiansen, 2007). The memo also allows the researcher to draw a mental line in the sand underneath a particular train of thought, to put it to one side, and then to re-direct their focus back to the data (Glaser, 1965). This helps to ensure that the theory is grounded in the data.

\section{Integrating categories and their properties}

This process builds on the constant comparisons described above but changes the items to be compared from one feature with another feature, to comparisons between features and elements of categories. Individual features are compared to an aggregated list of all the things that have been learnt about that category. So 'dungarees' are compared with all the characteristics of 'fun' - its playful, good humoured and lively nature. This served to bring the key aspects of the category into sharper relief. 
The categories also became integrated with each other: fun with warm, intellectual with highbrow; and it became clear the ways in which categories differ and are similar to each other. As one category was compared with another, the features of each category which were similar or different were identified. This analytical process leads inevitably to the development of a theory as linking themes emerge.

\section{Delimiting the theory}

The grounded theory analysis can be an overwhelming process for the analyst (Christiansen, 2007). As the items merge into categories and the categories into a theory, the confusion dissipates and a clarity emerges. One process which helps to speed this up is delimiting the theory. Early in the process of analysis a wide range of categories including control, interest in fashion and image consciousness had been identified. During the process of delimiting, it became clear that these were subsets of a higher level construct. A decision was therefore made to stop looking for new categories, and instead to focus on examples which would allow the existing categories to be refined.

\section{Writing the theory}

The fourth and final stage in Glaser's (1965) CGT is writing the theory. Glaser (1965) suggested that this should be started only when the researcher is convinced that the framework of theoretical codes and their elements gives a 'reasonably accurate' (p. 443) account of the phenomenon under scrutiny. The writing process involved collating the memos which were collected throughout the process of analysis, whilst continuing with the process of constant comparing the theory with the data, looking for evidence of theoretical gaps and seeking examples from the data which illustrate the elements of the theory.

\section{Findings}

We move now to a description of the grounded theory which was developed after close analysis of the data. The grounded theory developed offers a framework of occupational prototypes consisting of five intrapsychic and three interpsychic constructs (Haley, 1963/1990).

The intrapsychic constructs describe characteristics or elements of the individual which relate to their personality or character. These intrapsychic orientations stem from within the individual, but the symbols which represent them are 'co-created' within society. For example, warmth may be present in an individual regardless of their culture, but whether that warmth is expressed through (or symbolised by) a handshake, a hug, one/two/three or four kisses on the cheek will be influenced by the context.

The interpsychic constructs in contrast are entirely socially constructed, with both the construct itself and the symbol which represents it, co-created within society. The construct only exists within and with reference to society and the symbol is a culturally-specific manifestation of the relationship the individual has with their society. Being 'cool', for example, is a meaningful construct only within a social context - without a society, the notion that someone is 'cool' is meaningless. However, the tangible features which demonstrate whether someone is cool or not are culturally specific, varying from one culture or sub-culture 
to another. These interpsychic constructs thus are culturally constructed and symbolised by culturally specific features.

Figure 1 provides an overview of the framework.

Figure 1: A grounded theory of the characteristics of four occupational prototypes

[Figure 1 near here]

The constructs described in this framework were drawn from all the students' descriptions of their own prototypes of the occupations they described. Some of the occupations under scrutiny were more often linked to one construct than another. Prototypes of social workers, for example, were often warm; clinical psychologist prototypes were often highbrow. The prototypes of each occupation, however, varied considerably from one participant to another. For example, some primary teacher prototypes were conceptualised as fun, others were more serious; some occupational psychologist prototypes were warm and some much less so. The theory helps to explain the characteristics that participants used to conceptualise and discriminate between all of the prototypes, rather than describing the prototypical characteristics of any particular occupation. In the paragraph later that describes the 'energetic' construct, for example, the specific participant quotations which describe highenergy prototypes are drawn from some participants' descriptions of an organisational psychologist, a clinical psychologist and a primary teacher, and the quotations which describe low-energy prototypes are draw from other participants' descriptions of a social worker, a clinical psychologist and a primary teacher. The point is not how participants describe a clinical psychologist, but that when they imagine a typical clinical psychologist, they conceptualise this person in terms of their energy - whether that may be low or high. Each construct described as follows draws from the prototypes of all of the occupations described.

\section{Intrapsychic constructs}

\section{Warm}

The two codes that characterised the warm prototypes were setting considerable store by their close relationships, 'mine just has her arms full of kids'; and not being interested in projecting a particular image of themselves, 'there's lots of people and lots of busy-ness so it's kind of like there are many different elements and it's homely, it's warm, it's kind of full but it doesn't necessarily all fit together'. Prototypes who were not warm were described as detached and independent: 'There might be a relationship but if there is it's not very emotional, it's more kind of functional'; and image conscious, 'very sharp, very polished, very professional, [...] very high end'.

\section{Energetic}

The high energy orientated prototypes were characterised by three codes: their sociability, 'I also see that they do quite a lot of socialising', 'they have lots of friends'; the variety of their activities, 'I think there's lots of variety, lots of different places, different things to experience, different cultures, different people'; and the physical effort required for their hobbies, 'cycle to the South of France...skiing... rock climbing'. In contrast, those with lower levels of energy would be more likely to spend time on their own, 'they're all a bit introverted maybe and 
might read quietly or pet their cat', to go 'somewhere relaxing' on holiday; and in terms of pastimes: I'm just picturing sitting on the sofa sort of watching telly, having some dinner, just relaxing'.

\section{Fun}

The two codes which were integrated to form this construct were behaviour which could be considered frivolous rather than serious, and the desire for pleasure over meaning. The fun construct was illustrated through the clothes that the individual prototype might choose with those considered fun dressing 'quite informal, quite colourful, quite fun, like splodges of paint on the dungarees from helping the kids', in contrast to those who were more serious who 'just have the dull, like brown dull colours'. Choice of leisure activities were revealing, with those with high levels of fun engaging in 'fun kind of activities' such as karaoke or watching comedy programmes, and those who were considered more serious more likely to 'go to Athens and take themselves around and learn something, you know they'd be having an industrious holiday so like they're learning', or watch documentaries.

\section{Conventional}

The two codes to emerge from the data analysis of the conventional dimension were choosing the standard or classic option, and making the same choices that many others make. Prototypes with a conventional leaning were reported to make choices which might be considered the default: options which are classic, standard and chosen by many others, such as, choosing to wear 'maybe black and grey, with like kind of grey tights and a black skirt'. The more unconventional prototypes were reported to make choices which reflected their individualism and might be thought to be unusual, wearing an eye-catching accessory 'maybe with a more unconventional..., so for instance a big shawl'. Conventionally orientated prototypes might choose a package holiday or a city break, where the more unconventional 'they wouldn't do a package holiday, they would do their own thing'.

\section{Intelligent}

The key code which characterised prototypes with high levels of intelligence was that they sought out activities which made use of their intellect. They would watch educational programmes on television 'maybe science, more history, educational', and would read informative magazines; 'they might read the Economist and then they'll get Time magazine', or challenging fiction: 'intellectual fictional stuff-Booker prize winner kind of thing'. In contrast, the prototypes who were deemed less intelligent would watch television for entertainment such as Made in Chelsea, or soap operas: 'they watch Eastenders, and whatever other soaps' and would read magazines ('they'd probably get Grazia') and books ('romantic novels, chick lit') which deal with less weighty topics. The volume of reading that a prototype might engage with also symbolises intellectual status. Those with high levels of intelligence might enjoy reading as a pastime and their homes would be characterised by books and bookshelves: 'Lots of books in the house'.

\section{Interpsychic Constructs}

\section{Highbrow}


Symbols of cultural status revealed whether a prototype was thought to have interests and tastes which might be described as highbrow, or whether their choices were more middle or lowbrow. The prototypes with an orientation to highbrow culture would expose themselves to a range of cultural experiences and would enjoy learning about history, art and literature. For holidays they might enjoy a European city break - 'some place with cultural heritage', and might spend their weekends 'introducing the kids to museums'. They would choose cultural leisure activities ('maybe an art gallery') and their choice of red or white wine 'depends whether it's fish or meat'. Those with an orientation to low culture are more likely to opt for more popular activities. They might choose a beach holiday ('a beach with lots of people, you know a massive holiday resort)', and enjoy classic pub fare such as 'a burger and a pint'.

\section{Successful}

Prototypes with objective success were often symbolised by the status of their partner. A successful prototype would have a very desirable partner: good looking and well turned out, 'very attractive, very trendy and smart'; wealthy, 'her boyfriend's rich so he helps her out there'; and successful at work, 'a sort of intellectual with quite a high ranking post somewhere'. One key subset of objective success was money and symbols of money included the holidays the prototype might choose, 'mine has a place in the country and they go there quite a lot at weekends'; and the kinds of clothes they wear 'designer brands from head to toe' and shoes which are 'high end, expensive'.

\section{Cool}

Cool prototypes were stylishly dressed ('they're trendy, they've got some design and they've got a bit of colour') although not necessarily formally: 'mine is casual, he's in jeans, but they're very nice jeans, he's very trendy, and he's still got nice shoes on'. They were likely to live in particular parts of the city ('they're casual but they're quite stylish [...] they look like they come from like Clapham') and in fashionable homes: 'mine's in a flat in the East End in one of those renovated warehouses with the really big ceilings and the big windows and the ultra-trendy sort of environment'. Their leisure activities too added to their cool characters, with cool prototypes choose to go out 'up from a pub, so trendy, maybe gastro pubs' and opting for 'the cool places in town'.

These eight constructs then constitute our grounded theory, explaining the characteristics symbolised by the features of the four occupational prototypes described by these 24 psychology graduates. We will now present a discussion of the most notable aspects of the findings, situating them within the wider literature and will offer some tentative implications for practice.

\section{Discussion}

In this paper we present the results of a grounded theory study which explores the nature of occupational prototypes for a group of psychology undergraduates. Data from four focus groups provide myriad features that participants associated with four particular occupational prototypes. These features symbolise individual constructs, and the grounded theory proposes a framework of the attributes which are symbolised. The framework contains eight constructs, 
five intrapsychic constructs (warm, energetic, fun, conventional and intelligent) and three interpsychic constructs (highbrow, successful and cool). The discussion which follows offers an analysis of the findings of the study in the light of existing literature. In keeping with the recommended procedure for CGT (Glaser, 1965), some new, relevant literature will be introduced within the discussion below.

There are no existing theories of prototypical occupational identities, and no frameworks to account for the characteristics of people's perceptions of those who occupy certain occupations. Yet the elements described in this new theory, will be familiar to career researchers and counsellors. In the intrapsychic dimensions, overlaps could be discerned with aspects of the Five Factor model of personality (Costa \& McCrae, 1992), the Myers Briggs Type Indicator (Myers, 1962) and Holland's RIASEC framework (Holland, 1959). The interpsychic dimensions resonate with Bourdieu's capitals (Bourdieu, 1985) and with MacKinnon and Langford's framework of occupational prestige (1994).

Whilst these similarities are worth noting, this new theoretical framework is different from the existing taxonomies in that it focuses on perceptions rather than realities. From the perspective of those choosing career directions, this is important. Decisions are made on the basis of the individual's perceptions, and as such this constitutes an important angle for career research. In the discussion, therefore, we focus on the ways in which this theoretical framework extends the existing research on occupational prototypes, rather than focusing on an integration of the constructs with related but distinct literature.

As highlighted already, other studies have described the prototypes commonly associated with specific professions (Archer et al., 2010; Cheryan et al., 2013; Yates \& Cahill, 2018). Whilst previous literature describes prototypes of particular occupations, this study explores the nature of prototypes at a higher level of abstraction, examining the features of the prototypes, analysed as symbols (Blumer, 1969; Pratt \& Rafaelli, 1997, 2001). Existing research has explored the occupational prototypes of individual occupations, looking to identify and describe, for example, a prototypical computer scientist (Cheryan et al., 2013) or physicist (Packard \& Nguyen, 2003). These studies have aimed to find a shared description of each occupational identity, such as it is generally thought that scientists are likely to wear glasses. The analysis from this grounded theory study offers an overview of the characteristics which participants appeared to use to define and discriminate between occupational identities. Using descriptions of four occupational prototypes and identifying the characteristics which were symbolised by a range of features across all four prototypes, this framework highlights eight constructs which these participants used to help them to conceptualise their occupational prototypes.

This study then offers a theoretical framework which goes beyond a single occupation and a particular feature (scientists wear glasses). Rather than describing prototypes occupation by occupation, it offers an overview of the ways in which occupational prototypes in general are conceptualised (e.g. occupational prototypes are categorised on the basis of how intelligent they are). This responds to calls in the literature for a more explanatory analysis of the constructs, to complement the existing descriptive research (Kocoglu et al., 2016; Yates \& Cahill, 2018). It is possible that at this level of abstraction the theory could be generalisable to 
the prototypes of other occupations and in accordance with the grounded theory principle of modifiability (Holton, 2008), further theoretical exploration could lead to a theory which is relevant to a wider range of prototypical occupational identities and a wider range of individuals.

\section{The framework and career decision making}

This research has generated a starting point for a generalisable theory of occupational prototypes: a taxonomy which could represent the way people define and discriminate between occupational prototypes. Further research is clearly needed to modify, refine and explore the generalisability of this model, but in its current form it suggests that a formal theory of occupational prototypes could perhaps be developed (Strauss \& Corbin, 1990). This kind of model would not be tied to any individual's conceptualisation of the world of work, nor would it reflect the prototype of any single occupation, but would offer a more general set of categories that any individual could use to describe any occupational prototype. This then could offer a flexible tool that could be used by both researchers and practitioners to explore individual perceptions of occupations and their impact on career decision making.

We have reiterated that existing self-to-prototype matching research tends to focus on the degree to which an individual's self-concept aligns with a commonly shared prototype (Erlingher et al., 2018). We know that prototypes are made up of a combination of shared and individual features, yet research and practice which aims to identify the characteristics of each individual's occupational prototype, and measure the degree to which these align with their self-concept may be prohibitively complex. With a single framework that symbolises individual perceptions of the prototypes of different occupational groups, an exploration of individuals' perceptions becomes more manageable.

In this study we specifically aimed to allow participants to explore their prototypes as broadly and as in as much depth as possible, and in doing so, revealed that their conceptualisations of occupational identities incorporated significant and elaborate expressions of occupational identities outside work. The interview guide which was used to structure the focus groups encouraged the participants to conceptualise their prototypes outside the workplace, but also ensured that there was ample opportunity for the participants to describe work-related characteristics, activities and attitudes. It is notable that the participants chose not to focus discussions on the workplace itself, and that the relationships, behaviour and appearance of the prototypes they described were almost exclusively beyond the work context. Indeed, more than one participant commented explicitly that their knowledge of the job of an occupational psychologist was limited, and yet they found that they were able to describe their occupational psychologist prototype's lifestyle, appearance and values in some depth. It may be useful to reflect on the antecedents of these participants' prototypical features. How could a participant who reports having no real understanding of the job of an occupational psychologist still manage to build up a full image of their life in Clapham, the cocktail bars they go to after work, and their partner who works in the city? As mentioned already, people, particularly perhaps those making career choices at the start of their careers, in the absence of direct experience, will often make choices based on their perceptions of a particular career area. The focus observed in this study, on the lifestyle aspects of the occupational prototypes raises interesting 
questions about the influence that these ideas may have on career decisions. Further research could useful explore this in more depth.

The analysis, therefore, accounts for prototypical occupational identities holistically, focusing on perceptions of them outside work as well as within the workplace. This focus on the holistic nature of the prototypical identities reflects the acknowledgement in current career theory that career choices are influenced by and impact on people's lives overall (Savickas et al., 2009) and mirrors the growing evidence that boundaries between work and non-work are blurring (Cutts, Hooley \& Yates, 2015; Ramarajan \& Reid, 2013).

We have highlighted the contemporary paradigm in career development research which acknowledges the holistic nature of career choices and conceptualises choice about career as choices about identity (Ibarra \& Barbalescu, 2010; Meijers \& Lengelle, 2012). The grounded theory developed in this study seems to align with this approach. It suggests that not only do individuals who are making career choices understand themselves and their choices in terms of identity, but that identity thinking also contributes to their understanding of the world of work. This grounded theory suggests that as well as thinking about our career selves in terms of identities, we may also conceptualise occupations at least in part, in identity form.

\section{Practical implications for career counsellors}

The insights offered by this grounded theory explain nothing more than the phenomenon under study: the perceptions of four prototypical occupational identities described by 24 psychology undergraduates. As such, recommendations for practice are perhaps premature. Nevertheless, the findings hint at the promise of some new ideas for practice which may be useful to consider at this stage of theory development.

Contemporary career learning theories advocate that individuals should be supported to develop narratives about their future identities which can offer them some direction (Meijers \& Lengelle, 2012). A number of approaches have been put forward to help career counsellors to discuss identities with clients (e.g. Savickas, 2013; Savickas \& Hartung, 2012; Strauss, Griffin \& Parker, 2012) but, to date, no guidance is offered for career counsellors who want to discuss possible occupational identities. The findings from this study suggest that people may have occupation-related knowledge in the form of prototypes. According to Gottfredson's theory of circumspection and compromise (1981) and research on self-to-prototype matching (Hannover \& Kessels, 2004), information of this kind may have a significant influence on career choices and it is important that the knowledge be accurate, and that it is integrated with other aspects of their career learning. Indeed McPherson et al. (2018) have gone a stage beyond and have noted the importance of career decisions being made on accurate occupational information, demonstrating that inaccurate prototypes are associated with a less desirable assessment of an occupation. The framework here could form the starting point for a discussion about clients' preconceptions of particular occupational identities and how these might align with their own sense of identity.

The second practical implication concerns the blurring of work and non-work identities. Understanding an individual's identity as holistic is a well-established feature of contemporary career learning theories (Blustein, 2017; Savickas et al., 2013; Yates, 2016). The inclusion of 
non-work features in people's conceptualisations of occupations is less well documented but has been identified elsewhere in the literature (O'Dowd \& Beardslee, 1967; Pisarick, Rowell \& Currie, 2013; Yates \& Cahill, 2018). The findings of this study highlight the holistic nature of students' conceptualisations of occupational identities. This could suggest a new angle for exploration with clients, in which career counsellors invite clients to reflect on how their own identities might align with their perceptions of occupational identities in non-work as well as work contexts. Whilst some career counsellors might argue that issues of this nature should not feed into clients' career decisions, the evidence suggests that they do, and as such, discussions which expose and explore them could be of great value to clients.

Finally, it is interesting to reflect on the findings in this study with reference to the literature on stereotypes in career counselling. This literature focuses most often on gender stereotypes and to a lesser extent, on class and ethnicity (e.g. Archer et al., 2010; Gadassi \& Gati, 2009). The assumptions within this literature are that these stereotypes lead to limited career horizons and should be challenged in career conversations. The findings of the present study indicate that stereotype thinking focuses on much more than demographic characteristics, incorporating a range of individual characteristics. These prototypes may well be inaccurate and limiting and it would be important for career practitioners to uncover and challenge these preconceptions too.

\section{Limitations and directions for future research}

This grounded theory of prototypical occupational identities is based on the perceptions of four occupations, provided by 24 psychology students, all studying at the same institution in London. It offers a starting point for theory development rather than a finished product, and in accordance with grounded theory methodology, this theory should be modified in the light of future data (Holton, 2008). Further research which explores this arena with different participants and different occupations could provide valuable additional data which could be used to further develop this theory.

As this was an exploratory study, focus groups were chosen as an approach to data collection, as they allow for the efficient collection of considerable amount of data, but there are limitations associated with this approach. In particular, focus groups can lead to a tendency for the data to be homogenised, as participants with more extreme views are less likely to vocalise their opinions in front of other participants. Further research could make use of a wider range of approaches to data collection.

This study did not set out to focus on gender, but the population from which participants were selected (undergraduate students studying for a BSc in psychology) was predominately female and whilst all undergraduates students, male and female, were invited to take part, only women chose to participate in the research. It is plausible that the particular features of the occupational prototypes depicted by the participants were linked to their gender, and quite possible that a more mixed group may have identified a different range of features. For example, whilst participants were asked to explore both the outfits worn by their prototypes and the cars they might drive, the focus group discussions lingered longer on fashion than on vehicles; it is possible that this choice was a result of the feminine proclivities of the participant group, and that an all-male group may have taken the discussion in a different direction. 
Whether other areas of discussion would have generated features which symbolised different constructs is a second question, and future research should explore both the features and characteristics symbolised by those features, for a different group of participants.

\section{Conclusion}

This study provides a theoretical framework of prototypical occupational identities. The grounded theory conceptualises the features of occupational prototypes as symbols, and, abstracting this information, offers a framework of eight constructs (highbrow, successful, cool, warm, energetic, fun, intelligent and conventional) which are symbolised by the features of the occupational prototypes described by the psychology students in this study. As a substantive grounded theory, the theory is closely based on the data gathered in this study and constitutes a workable and modifiable theory. The theory reflects ideas within contemporary career literature about the importance of identities and the holistic nature of identities and career decisions. The theory of occupational prototypes may offer a new approach to identity-based career counselling but further development of the theory is needed. 


\section{References}

Archer, L., DeWitt, J., Osborne, J., Dillon, J., Willis, B., \& Wong, B. (2010). "Doing" science versus "being" a scientist: Examining 10/11-year-old schoolchildren's constructions of science through the lens of identity. Science Education, 94(4), 617-639.

Bagnoli, A., \& Clark, A. (2010). Focus groups with young people: a participatory approach to research planning. Journal of youth studies, 13(1), 101-119.

Blumer, H. (1969). Symbolic Interactionism: Perspective and Method. Englewood Cliffs, NJ: Prentice-Hall.

Blustein, D. L. (2017). The psychology of working: A New Perspective for Career Development. Career Planning \& Adult Development Journal, 33(2), 60-68.

Bourdieu, P. (1985). The market of symbolic goods. Poetics, 14(1-2), 13-44.

Bowen, G.A. (2005). Preparing a qualitative research-based dissertation: lessons learned. The Qualitative Report 10(2), 208 - 222.

Boychuk, C., Lysaght, R., \& Stuart, H. (2018). Career Decision-Making Processes of Young Adults With First-Episode Psychosis. Qualitative health research, 28(6), 1016-1031.

Castelli, L., Carraro, L., Tomelleri, S., \& Amari, A. (2007). White children's alignment to the perceived racial attitudes of the parents: Closer to the mother than the father. British Journal of Developmental Psychology, 25(3), 353-357.

Charmaz, K. (2013). Grounded theory methods in social justice research. In N.K. Denzin \& Y.S. Lincoln (eds), Strategies of qualitative inquiry, Thousand Oaks, US: Sage, pp291-336.

Charmaz, K., \& Henwood, K. (2017). Grounded theory methods for qualitative psychology. In C. Willig \& W. Stainton-Rogers (eds), The SAGE handbook of qualitative research in psychology, Palo Alto, US: Sage, pp. 238-260.

Charon, J.M. (2010). Symbolic Interactionism: An introduction, an interpretation, an integration Boston MA: Prentice Hall.

Cheryan, S., \& Plaut, V. C. (2010). Explaining underrepresentation: A theory of precluded interest. Sex roles, 63(7-8), 475-488.

Christiansen, O. (2007). A simpler understanding of classic GT: How it is a fundamentally different methodology. The Grounded Theory Review, 6(3), 39-61.

Clark, R., \& Lang A. (2002). Blanacing Yin and Yang: Teaching and Learning Qualitative Data Analysis within an Undergraduate Quantitative Analysis Course Teaching Sociology, 30(3), 343 - 360 . 
Cheryan, S,. Plaut, V.C., Handron, C. \& Hudson, L. (2013). The stereotypical computer scientist: gendered media representations as a barrier to inclusion for women Sex Roles 69(1), 58-71.

Costa, P. T., \& McCrae, R. R. (1992). Four ways five factors are basic. Personality and individual differences, 13(6), 653-665.

Crooks, D. L. (2001). The importance of symbolic interaction in grounded theory research on women's health. Health Care For Women International, 22(1/2), 11-27.

Cutcliffe, J. R. (2003). Reconsidering reflexivity: Introducing the case for intellectual entrepreneurship. Qualitative health research, 13(1), 136-148.

Cutts, B, Hooley, T. and Yates, J. (2015) Fitting in or being yourself? How undergraduates plan to use hair, clothes and make-up to smooth their transition to the workplace. Industry and Higher Education, 29(4), 271-282.

Davis, L.E. (1996). Learning qualitative research: Electronic Learning Circles Qualitative Health Research 6(3), $453-357$.

Ehrlinger, J., Plant, E. A., Hartwig, M. K., Vossen, J. J., Columb, C. J., \& Brewer, L. E. (2018). Do Gender Differences in Perceived Prototypical Computer Scientists and Engineers Contribute to Gender Gaps in Computer Science and Engineering?. Sex roles, 78(1-2), 40-51.

Fernandez, C. (2012) Guest Editorial, Themed Section. The Grounded Theory Review, 11(1), 7 28.

Gadassi, R., \& Gati, I. (2009). The effect of gender stereotypes on explicit and implicit career preferences. The Counseling Psychologist, 37(6), 902-922.

Glaser, B. G. (1965). The constant comparative method of qualitative analysis. Social problems, 12(4), 436-445.

Glaser, B. G. (1978). Theoretical sensitivity: Advances in the methodology of grounded theory. Sociology Press.

Glaser, B. G. (1992). Basics of grounded theory analysis: Emergence vs forcing. Sociology Press. Glaser, B. G. \& Strauss, A. L. (1967). The Discovery of Grounded Theory: Strategies for Qualitative Research, Chicago: Aldine Publishing Company.

Gottfredson, L.S. (1981). Circumscription and compromise: A developmental theory of occupational aspirations Journal of Counseling Psychology, 28, 545-579.

Gottfredson, L. S. (2002). Gottfredson's theory of circumscription, compromise, and selfcreation. In D. Brown (Ed.) Career choice and development (pp. 85-148). San Francisco: JosseyBass. 
Gough, B., Fry, G., Grogan, S., \& Conner, M. (2009). Why do young adult smokers continue to smoke despite the health risks? A focus group study. Psychology and Health, 24(2), 203-220.

Guichard, J. (2005). Life-long self-construction. International Journal for Educational and Vocational Guidance, 5(2), 111-124.

Haley, J. (1990). Strategies of psychotherapy (2nd edn). Rockville, MD: Triangle Press. (Original work published 1963).

Hannover, B., \& Kessels, U. (2004). Self-to-prototype matching as a strategy for making academic choices. Why high school students do not like math and science. Learning and instruction, 14(1), 51-67.

Haslam, S. A., \& Wilson, A. (2000). In what sense are prejudicial beliefs personal? The importance of an in-group's shared stereotypes. British Journal of Social Psychology, 39(1), 4563.

Hernandez, C. A. (2008). Are there two methods of grounded theory? Demystifying the methodological debate. The Grounded Theory Review, 7(2), 39-66.

HESA (2014). Graduate Destinations. Cheltenham: Higher Education Statistics Authority. Retrieved from https://www.hesa.ac.uk/data-and-analysis/students/destinations

Hilton, J. L., \& von Hippel, W. (1996). Stereotypes. Annual Review Of Psychology, 47(1), 237 271.

Holland J. (1959). A theory of vocational choice. Journal of Counseling Psychology, 6(1), 35 45.

Holton, J.A. (2007). The Coding Process and its Challenges. In A. Bryant \& K. Charmaz (Eds) The SAGE Handbook of Grounded Theory (pp.265 - 389). Thousand Oaks CA: Sage.

Hussein, M. E., Hirst, S., Salyers, V., \& Osuji, J. (2014). Using Grounded Theory as a Method of Inquiry: Advantages and Disadvantages. The Qualitative Report, 19(27), 1-15

Hyde, A., Howlett, E., Brady, D., \& Drennan, J. (2005). The focus group method: Insights from focus group interviews on sexual health with adolescents. Social Science and Medicine, 61(12), 2588 - 2599. 
Ibarra, H., \& Barbulescu, R. (2010). Identity as narrative: Prevalence, effectiveness, and consequences of narrative identity work in macro work role transitions. Academy of management review, 35(1), 135-154.

Jung, J. Y., \& Young, M. (2019). The occupational/career decision-making processes of intellectually gifted adolescents from economically disadvantaged backgrounds: A mixed methods perspective. Gifted Child Quarterly, 63(1), 36-57.

Jussim, L., Cain, T. R., Crawford, J. T., Harber, K., \& Cohen, F. (2009). The unbearable accuracy of stereotypes. In, T.D. Nelson (Ed.), Handbook of prejudice, stereotyping, and discrimination, (pp. 199-227). Hove: Psychology Press.

Kitzinger, J. (1994). The methodology of focus groups: the importance of interaction between research participants. Sociology of health \& illness, 16(1), 103-121.

Koçoğlu, I., Akgün, A. E., \& Keskin, H. (2016). The Collective Unconscious at the Organizational Level: The Manifestation of Organizational Symbols. Procedia-Social and Behavioral Sciences, 235(24), 296-303.

Krueger, R. A. (1994). Focus groups: a practical guide for applied research. 1994. Thousands Oak California: SAGE Publications Inc.

Lippmann W. (1991). Public opinion. New Brunswick, NJ: Transaction Publishers. Original work published 1922.

Lowe, A. (2005). Trust in emergence Keynote presentation delivered to the $3^{\text {rd }}$ International qualitative Research convention University Teknologi, Malaysia.

MacKinnon, N. J., \& Langford, T. (1994). The meaning of occupational prestige scores: A social psychological analysis and interpretation. The Sociological Quarterly, 35(2), 215-245.

Mastro, D. E., Behm-Morawitz, E., \& Kopacz, M. A. (2008). Exposure to television portrayals of Latinos: The implications of aversive racism and social identity theory. Human Communication Research, 34(1), 1-27.

McPherson, E., Park, B., \& Ito, T. A. (2018). The Role of Prototype Matching in Science Pursuits: Perceptions of Scientists That Are Inaccurate and Diverge From Self-Perceptions Predict Reduced Interest in a Science Career. Personality and Social Psychology Bulletin, 44(6), 881898.

Meijers, F., \& Lengelle, R. (2012). Narratives at work: The development of career identity. British Journal of Guidance \& Counselling, 40(2), 157-176. 
Myers, I. B. (1962). The Myers-Briggs Type Indicator: Manual. Palo Alto US: Consulting Psychologists Press.

O'Dowd, D. D., \& Beardslee, D. C. (1967). Development and consistency of student images of occupations. Rochester, US: Michigan State University.

Packard, B. W. L., \& Nguyen, D. (2003). Science career-related possible selves of adolescent girls: A longitudinal study. Journal of Career Development, 29(4), 251-263.

Peters, K., Ryan, M, Haslam, S.A. \& Fernandes, H. (2012). To belong or not to belong: evidence that women's occupational disidentification is promoted by lack of fit with masculine occupational prototypes, Journal of Personnel Psychology 11(3), 148-158.

Pisarik, C. T., Rowell, P. C., \& Currie, L. K. (2013). Work-related daydreams: A qualitative content analysis. Journal of Career Development, 40(2), 87-106.

Pratt, M. G., \& Rafaeli, A. (1997). Organisational dress as a symbol of multilayered social identities. Academy of Management Journal, 40(4), 862-898.

Pratt, M. G., \& Rafaeli, A. (2001).Symbols as a language of organizational relationships. Research in Organizational Behavior, 23, 93-132.

Ramarajan, L., \& Reid, E. (2013). Shattering the myth of separate worlds: negotiating nonwork identities at work. Academy Of Management Review, 38(4), 621-644.

Rosch, E., \& Mervis, C. B. (1975). Family resemblances: Studies in the internal structure of categories. Cognitive psychology, 7(4), 573-605.

Ryan, M. (2014). Who is Like a Scientist? A Self-Prototype Matching Approach to Women's Underrepresentation in STEM Fields (Doctoral dissertation, University of Washington).

Savickas, M. L. (2013). Career construction theory and practice. In S.D. Brown \& R.W. Lent (eds) Career development and counseling: Putting theory and research to work, ( $2^{\text {nd }}$ edition) pp144-180. Wiley.

Savickas, M.L. (2015). Career counseling paradigms: Guiding, developing, and designing. In P. J. Hartung, M. L. Savickas and B. W. Walsh (Eds), APA handbook of career intervention (pp. 129143). Washington, DC, US: American Psychological Association.

Savickas, M. L., \& Hartung, P. J. (2012). My career story. An autobiographical workbook for lifecareer success. Presentation at the annual meeting of the National Career Development Association.

Savickas, M. L., Nota, L., Rossier, J., Dauwalder, J. P., Duarte, M. E., Guichard, J., ... \& Van Vianen, A. E. (2009). Life designing: A paradigm for career construction in the 21st century. Journal of vocational behavior, 75(3), 239-250.

Schneider, D. J. (2004). The psychology of stereotyping. New York: Guilford Press. 
Schreiber, R.S. (2001). The "How To" of Grounded Theory, Avoiding the Pitfalls. In R.S. Schreider, \& P.N. Stern (eds), Using grounded theory in nursing. Philadelphia, US: Springer.

Sherriff, N., Gugglberger, L., Hall, C. and Scholes, J. (2014). "From start to finish": practical and ethical considerations in the use of focus groups to evaluate sexual health services interventions for young people. Qualitative Psychology 1(2), 92- 106.

Sim, J. (1998). Collecting and analysing qualitative data: issues raised by the focus group. Journal of Advanced Nursing, 28(2), 346 - 352.

Starr, C. R. (2018). "I'm Not a Science Nerd!" STEM Stereotypes, Identity, and Motivation Among Undergraduate Women. Psychology of Women Quarterly, 42(4), 489-503.

Strati, A. (1998). Organizational symbolism as a social construction: A perspective from the sociology of knowledge. Human Relations, 51(11), 1379-1402.

Strauss, A., \& Corbin, J. (1990). Basics of Qualitative Research - Techniques and Procedures for Developing Grounded Theory, $\left(2^{\text {nd }}\right.$ ed). Thousand Oaks, CA: Sage.

Strauss, K., Griffin, M. A., \& Parker, S. K. (2012). Future work selves: How salient hoped-for identities motivate proactive career behaviors. Journal of Applied Psychology, 97(3), 580 - 598.

Tolich, M. (2009). The principle of caveat emptor: confidentiality and informed consent as endemic ethical dilemmas in focus group research. Journal of Bioethical Inquiry, 6(1), 99 - 108.

Wade, P., Bergeron, C., White, K., Teeman, D., Sims, D. \& Mehta, P. (2010). Key Stage 2 CareerRelated Learning Pathfinder Evaluation. London: Department for Education.

Warr, D. J. (2005). "It was fun... but we don't usually talk about these things": analyzing sociable interaction in focus groups. Qualitative Inquiry, 11(2), 200-225.

Willig, C. (2013). Introducing qualitative research in psychology. McGraw-Hill Education (UK).

Yates, J. (2017). A meta-theoretical framework for career practitioners. The Indian Journal of Career and Livelihood Planning, 5(1), 15-25.

Yates, J. \& Cahill, S. (2018). What kind of shoes does a social worker wear? A content analysis of four occupational prototypes. British Journal of Guidance and Counselling. Online first 10.1080/03069885.2018.1437596 


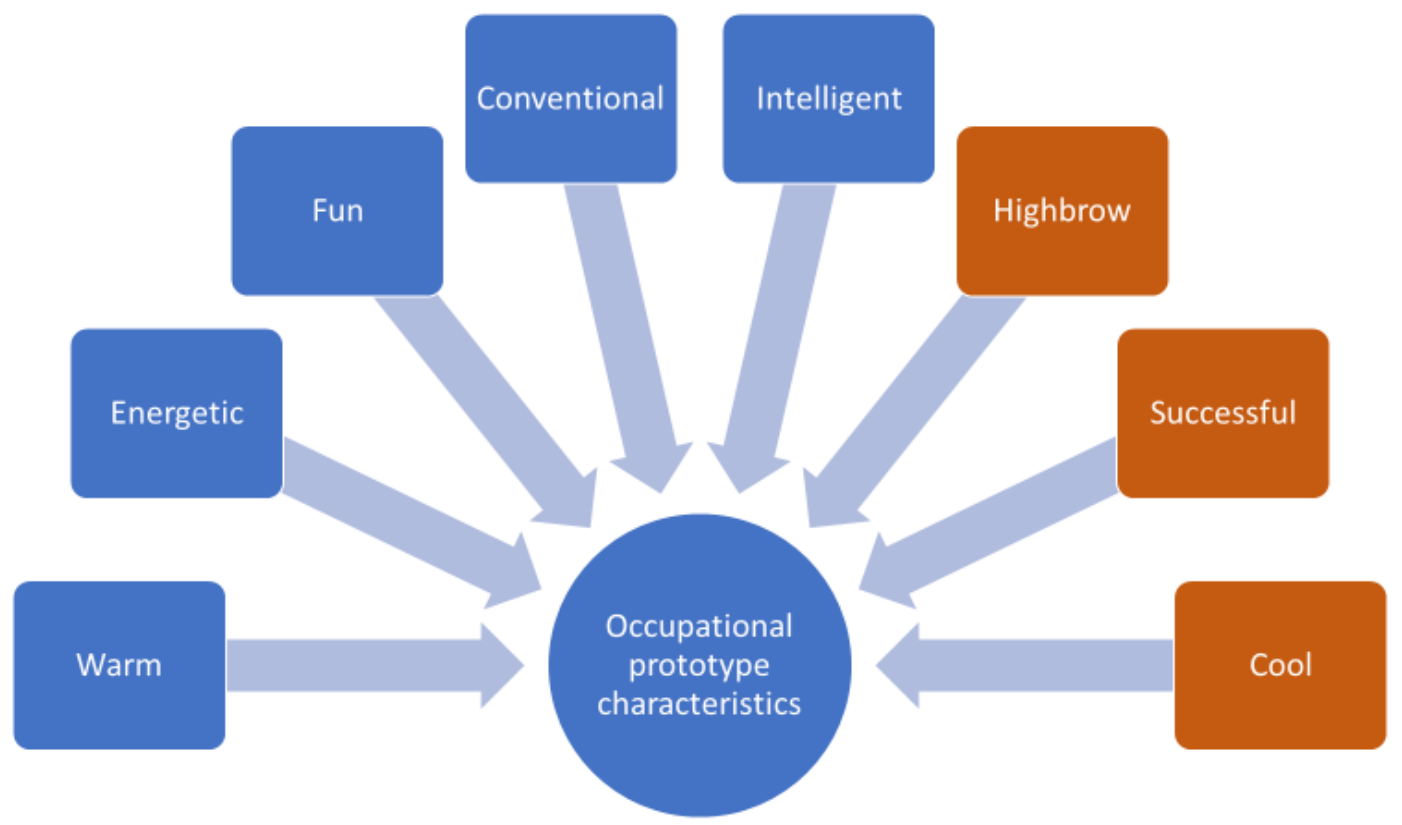

Figure 1: A grounded theory of the characteristics of four occupational prototypes 